\title{
Characterization of prostate cancer cell progression in zebrafish xenograft model
}

\author{
WEI XU ${ }^{1,2}$, BRITTANY A. FOSTER ${ }^{1,3}$, MACKENZIE RICHARDS ${ }^{1,2}$, \\ KENNETH R. BONDIOLI ${ }^{1,3}$, GIRISH SHAH ${ }^{4}$ and CHRISTOPHER C. GREEN ${ }^{1,2}$ \\ ${ }^{1}$ Louisiana State University Agricultural Center; ${ }^{2}$ School of Renewable Natural Resources, \\ Louisiana State University; ${ }^{3}$ School of Animal and Food Sciences, Louisiana State University, \\ Baton Rouge, LA 70808; ${ }^{4}$ School of Pharmacy, University of Louisiana, Monroe, LA 71201, USA
}

Received August 11, 2017; Accepted October 13, 2017

DOI: $10.3892 /$ ijo.2017.4189

\begin{abstract}
Early diagnosis of prostate cancer (PCa) is critical for the application of efficient treatment to $\mathrm{PCa}$ patients. However, the majority of PCas remains indolent from several months to several years before malignancy. Current diagnosis methods have limitations in their reliability and are inefficient in time cost. Thus, an efficient in vivo PCa cell xenograft model is highly desired for diagnostic studies in PCas. In the present study we present a standardized procedure to create a $\mathrm{PCa}$ cell xenograft model using zebrafish (Danio rerio) as the host. PC3-CTR cells, a cell line from adenocarcinoma with stable expression of calcitonin receptor (CRT), were subcutaneously injected into zebrafish larvae at $48 \mathrm{~h}$ post fertilization. The nursing conditions for the larvae were optimized with stable survival rates of post hatch and post PC3-CTR cell injection. In this system, the progression of PC3-CTR cells in vivo was evaluated by migration and proliferation of the cells. Massive migrations of PC3 cells in vivo were observed at post injection day (PID)3. The injected PC3-CTR cells eventually invaded the whole larval zebrafish at PID5. Quantification of PC3-CTR cell proliferation was done using quantitative PCR (qPCR) analysis targeting the expression profiles of two PCa housekeeping genes, TATA-binding protein (TBP) and hypoxanthine phosphoribosyltransferase 1 (HPRT1) encoding genes. The excessive proliferation of PC3 cells in vivo was detected with both qPCR assays. Expression levels of one non-coding gene, prostate cancer associated 3 gene ( $p c a 3)$, and two other genes encoding transient receptor potential ion channel Melastatin 8 (trpm8) and prostate-specific membrane antigen (psma), showed a significantly enhanced aggressiveness of PC3-CTR cells in vivo. The model established in the present study provides an improved in vivo model for the diagnosis of PCas efficiently. This PCa cell xenograft model
\end{abstract}

Correspondence to: Professor Wei Xu, Louisiana State University Agricultural Center, 2410 Ben Hur Road, Baton Rouge, LA 70820, USA

E-mail:wxu@agcenter.lsu.edu

Key words: prostate cancer, xenograft, zebrafish can also serve as a tool for high throughput anti-PCa drug screening in therapeutic treatments.

\section{Introduction}

As the second leading cause of cancer-related deaths in American men (1), prostate cancer (PCa) typically undergoes slow growth and often leaves the patient susceptible to other diseases during the development. In a significant subset of men, the cancer develops rapidly, leading to death (2). Depending on the characteristics of PCas, different treatment strategies are available to the patients. The indolent PCas in patients usually do not require major treatment, while rapid developing PCas require aggressive treatments to prevent further malignancy. Therefore, it is critical to detect the aggressive PCas at their early stages. Recent studies suggest that most of the PCas are initiated by a small population of tumor-initiating cells, which are responsible for growth of the tumor, metastasis and even relapse (3). Differentiation of these cells leads to a high diversity of tumor cell populations $(4,5)$. Characterization of tumor-initiating cells provides important information for the prediction of future clinical behavior of the tumor.

The diagnosis of early $\mathrm{PCa}$ in patients has been commonly studied for many years. However, many strategies have been developed in the past few decades. Some PCa specific DNA markers have been identified and applied to the detection of PCas in elderly men $(6,7)$. Although these markers can rapidly provide a reference for the risk of PCas in humans, they cannot predict whether the tumor will be a slow-growing or an aggressive one that needs immediate treatment. A sufficient animal model that allows the human prostate tumor cells to grow and develop normally, and demonstrates the fate of tumor cells in a short time period is highly desired.

The classic approach to assess tumorigenicity and metastatic potential of human prostate cells is the growth of orthotopic xenografts in immune-deficient mice (8-10). The model has been extensively used in functional analyses of $\mathrm{PCa}$ related genes as well as drug screening for PCa treatments. However, the development of tumor xenografts from prostate cancer cells implanted in mouse prostate tissue usually takes two months or longer. This is not the optimal period for the diagnosis of aggressiveness of a PCa in clinical studies. 
a

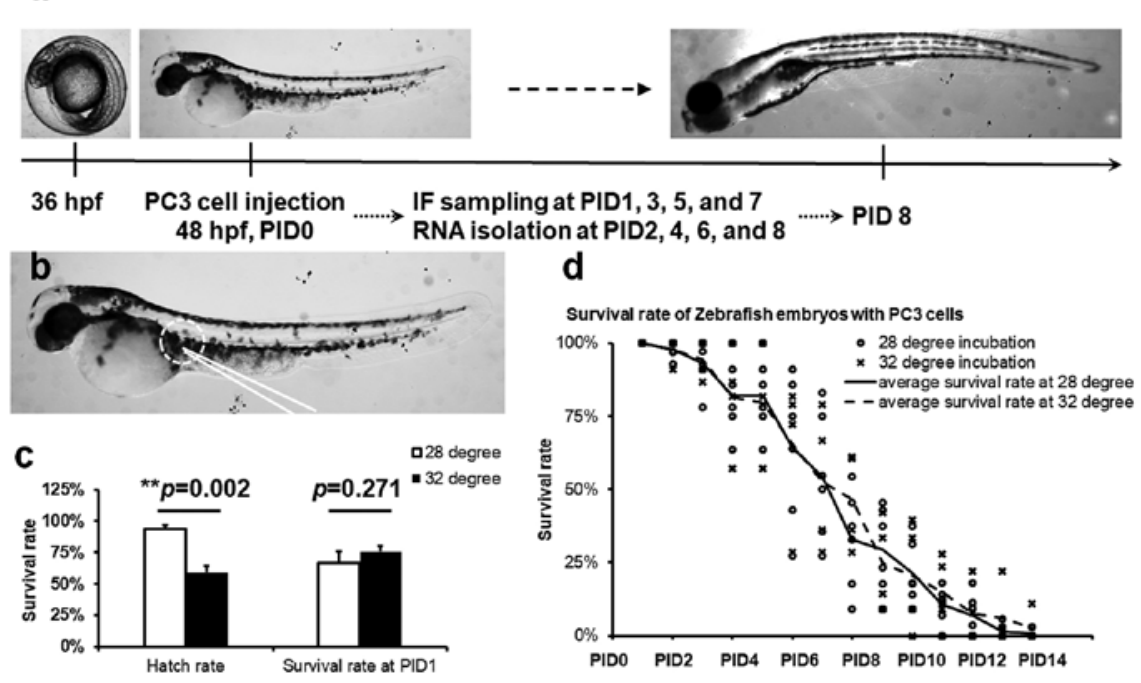

Figure 1. Experimental design and conditions for zebrafish xenograft model. (a) The PC3-CRT cells were introduced subcutaneously to zebrafish embryos at $48 \mathrm{~h}$ post fertilization (hpf) followed by an incubation at $28(\mathrm{n}=350)$ or $32^{\circ} \mathrm{C}(\mathrm{n}=277)$. (b) The microinjection site in zebrafish embryo is indicated with white circle. (c) The hatching rates of zebrafish embryos and survival rates of zebrafish larvae at PID1 incubated at 28 ( $\mathrm{n}=318$ ) or $32^{\circ} \mathrm{C}(\mathrm{n}=169$ ). (d) Survival rates of zebrafish larvae implanted with PC3 cells during post injecting nursing at $28(\mathrm{n}=318)$ or $32^{\circ} \mathrm{C}(\mathrm{n}=169)$. PID, post injection day; IF, immunofluorescence.

Additionally, this model is costly and labor intensive, which is a disadvantage in high throughput anticancer drug screenings.

Recent studies have shown that zebrafish (Danio rerio) can serve as a useful model organism for cancer research (11-14). Zebrafish offer several unique advantages over mammalian models, such as small animal size, no extensive surgical procedures, strong reproducibility and rapid growth of xenografts (15). Moreover, the translucent body of the fish embryos enables us to visualize all processes associated with tumor formation, tumor progression, metastasis and death (16). Furthermore, the zebrafish is more xenograft-tolerant and has less immune response to tumor cell xenograft than mice (17). Another major advantage is that an extremely small number cells are required for implantation (as little as $1 \times 10^{3}$ cells). Thus, even primary prostate cells derived from tumor biopsies can be sufficient for this model.

In the present study, we established the human prostate tumor cell zebra fish xenograft model using a well-defined human prostate cancer cell line, PC3-CTR. Previously created in Dr Shah's laboratory, the cell line was transfected with a pcDNA3.1 vector carrying the full-length CTR cDNA (18). Unlike the wild-type PC3 cell line, the PC3-CTR can stably express calcitonin receptor, which enhances the aggressiveness of the cells (18). The goal of this study is to examine the process of tumor growth in zebrafish embryos, and monitor the expression of some prostate cancer-specific markers during this process. The conditions of zebrafish embryos implanted with PC3-CTR cells were optimized and the migration of implanted PC3-CTR cells in zebrafish embryos were monitored using live cell fluorescent dyes. The proliferation and development of the PC3-CTR cells in zebrafish embryos were also evaluated using $\mathrm{PCa}$ cell markers.

\section{Materials and methods}

Generation of zebrafish embryos. All experiments on zebrafish were performed in compliance with the Louisiana State
University, Agricultural Center's (LSU AgCenter) institutional guidelines and under the National Research Council's criteria for humane care as outlined in the 'Guide for the Care and Use of Laboratory Animals'. The protocol used in the present study was approved by the LSU AgCenter Institutional Animal Care and Use Committee.

Sexually matured zebrafish individuals were maintained in the Aquaculture Research Station at the LSU AgCenter. Adult zebrafish were spawned in water at $28^{\circ} \mathrm{C}$ on a 14 -h light $/ 10-\mathrm{h}$ dark cycle. The procedures for breeding, embryo collection, staging (Fig. 1a) and rearing all followed previously published standard protocols (19).

Microinjection of prostate cancer cell line to zebrafish embryos. A prostate cancer cell line, PC3-CTR was used for microinjection. The cells were cultured overnight at $37^{\circ} \mathrm{C}$ with $5 \% \mathrm{CO}_{2}$ supply in the RPMI-1640 medium (Corning Life Sciences, Corning, NY, USA) supplemented with $10 \mathrm{mM}$ HEPES, $25 \mathrm{mM} \mathrm{NaHCO}$, 10\% fetal bovine serum (FBS; VWR/Life Science, Radnor, PA, USA), $200 \mathrm{mg} / 1$ geneticin (VWR/Life Science), $100 \mu \mathrm{g} / \mathrm{ml}$ penicillin $\mathrm{G}$, and $100 \mu \mathrm{g} / \mathrm{ml}$ streptomycin (100x Amresco penicillin/streptomycin stocking solution from VWR/Life Science). Thereafter, the cells were incubated with a live-cell imaging dye Qtracker ${ }^{\circledR} 525$ (Thermo Fisher Scientific, Waltham, MA, USA) for $1 \mathrm{~h}$ at $37^{\circ} \mathrm{C}$ to be fluorescently labeled. The labeled PC3-CTR cells were digested by $0.05 \%$ Trypsin (Corning Life Sciences) and re-suspended in phosphate-buffered saline (PBS; Corning Life Sciences). The cell density in the suspension was calculated based on cell counting with a hemocytometer and was adjusted to 500 cells $/ \mathrm{ml}$ in PBS.

The zebrafish embryos at $48 \mathrm{~h}$ post fertilization (hpf) were manually dechorionated, and anesthetized with $10 \mu \mathrm{g} / \mathrm{ml}$ tricaine methanesulfonate (MS-222; Sigma-Aldrich, St. Louis, MO, USA) and transferred to $50 \mu 1$ drops of filtered aquarium water with $10 \mu \mathrm{g} / \mathrm{ml} \mathrm{MS-222} \mathrm{under} \mathrm{oil} \mathrm{in} \mathrm{a} 5-\mathrm{cm}$ diameter petri-dish, also containing a $20 \mu \mathrm{l}$ drop of PC3-CTR cells 


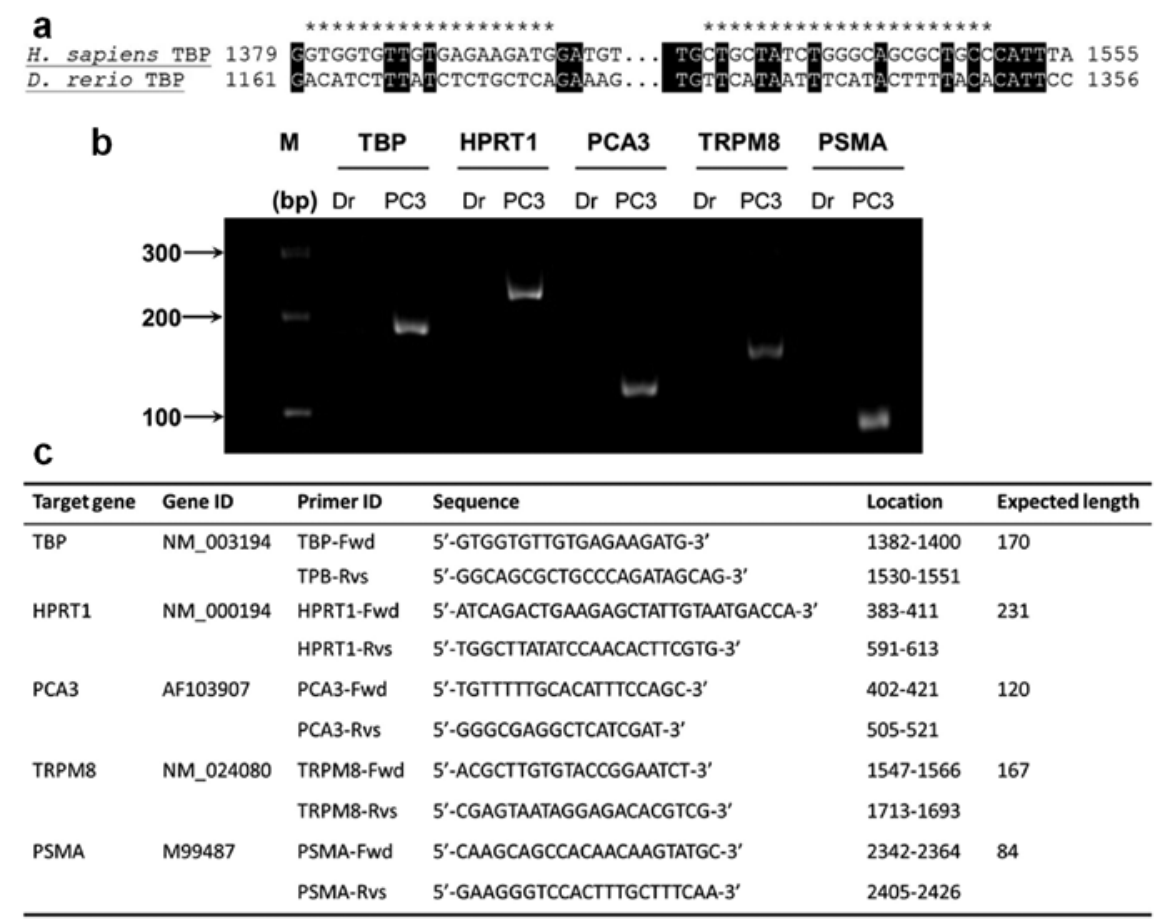

Figure 2. Human specific PCR primers for molecular markers. (a) Locations of human $t b p$ specific primers were selected based on the alignment of human and zebrafish $t b p$ genes. The targeting sequences of forward and reverse primers are marked with an '*'. (b) The specificities of synthesized primers were analyzed with qPCR and electrophoresis on PAGE-TBE gel. PCR products of all five pairs of primers were only seen in human PC3-CRT cells (PC3) not in zebrafish samples (Dr). (c) The primer information is listed. M, DNA marker.

diluted in PBS suspension. Microinjection was performed with an Eppendorf CellTram microinjection system, on a Nikon Eclipse TE200 inverted microscope. Cells were back-filled into the microinjector (22 $\mu \mathrm{m}$ ID) and five to six PC3-CTR cells were injected subcutaneously through the sinus venosus above the yolk (Fig. 1B). The embryos were immediately transferred to fresh filtered aquarium water and incubated at $37^{\circ} \mathrm{C}$ for $2 \mathrm{~h}$. Following, the embryos were maintained at 32 or $28^{\circ} \mathrm{C}$ for up to 12 days post transplantation (14 days post fertilization).

Larval zebrafish maintenance and sampling. Starting from post-hatching day 4, freshly hatched Artemia (Pentair plc, Sanford, NC, USA) were produced daily and used twice a day as diet for larval zebrafish. Growth of the larval zebrafish was monitored daily using a stereomicroscope. The development of PC3-CTR cells in zebrafish was visualized using a Nikon Eclipse Ti fluorescent microscope (Nikon USA, Melville, NY, USA) following the anesthesia of the larvae with $50 \mu \mathrm{g} / \mathrm{ml}$ MS-222 (Thermo Fisher Scientific). PC3-CTR cells in zebrafish embryos were visualized under fluorescent microscope. The PC3-CTR cells were monitored daily and mortalities of zebrafish were recorded. Some surviving zebrafish larvae were harvested for immunofluorescent (IF) staining or RNA isolation. Larvae for IF were first anesthetized with MS-222 as described above and fixed with $4 \%$ paraformaldehyde (PFA) overnight at $4^{\circ} \mathrm{C}$. Then, they were preserved in $10 \%$ methanol at $4^{\circ} \mathrm{C}$. Some anesthetized larvae were immediately transferred to Tri-Reagent ${ }^{\circledR}$ for RNA isolation.

Immunofluorescent staining of human specific antigen. The PFA fixed larval samples in methanol were rehydrated with a serially diluted methanol in PBS with the concentrations, 100, 75 and $50 \%$. After washing with PBS, each sample was blocked with $10 \%$ goat serum in PBS for $2 \mathrm{~h}$. Thereafter, the sample was incubated with mouse anti-human nuclei antibody (EMD Millipore, Billerica, MA, USA) in PBS (1:1,000 dilution) at $4^{\circ} \mathrm{C}$ overnight. A secondary antibody (goat $\mathrm{x}$ mouse) fluorescently labeled with Alexa 594 (Thermo Fisher Scientific) was diluted with PBS at 1:500 and incubated with the larval sample at room temperature for $1 \mathrm{~h}$ in a dark environment. The sample was observed under the Nikon Eclipse Ti fluorescent microscope (Nikon USA).

Quantification and characterization of PC3-CTR cells in larval zebrafish using quantitative PCR. To estimate the number of PC3-CTR cells in each zebrafish larval individual, we developed a quantitative PCR (qPCR) assay targeting housekeeping genes encoding TATA-binding protein (TBP) and hypoxanthine phosphoribosyltransferase 1 (HPRT1) (20-22) in PC3-CTR cells. The full sequences of human tbp and hprt1 were downloaded from GenBank database and used for homologues searching through zebrafish nucleotide database using BLASTn (23). No homologues of human hprtl was found in Danio rerio database, while a highly conservative tbp gene was found in Danio rerio database. Therefore, the qPCR primers specific for human hprtl were designed by the primer designing tool on IDT DNA (Coralville, IA, USA) website, while the primers for human $t b p$ were designed based on a high variance region in the sequence compared to Danio rerio tbp (Fig. 2a). The specificity of the qPCR primers was tested by PCR with cDNAs from PC3-CTR and zebrafish larvae and the PCR products were visualized by electrophoresis with $7 \%$ acrylamide-bisacrylamide TBE gel (Fig. 2b). 
A PC3-CTR cell number against qPCR Ct value standard curves were created based on the qPCR amplification profiles of human tbp and hprtl. Briefly, the PC3-CTR cells were harvested from a subconfluent cell culture plate and quantified with a hemocytometer under a microscope following the standard protocol. Each zebrafish larval individual at different development stages (from day 1 to day 12 post hatching) without PC3-CTR implantation was mixed with a certain number of PC3-CTR cells diluted with PBS. The ratios of larva and PC3-CTR numbers are, 1:5, 1:50, 1:500, 1:5,000, 1:50,000 and 1:500,000. For example, for the dataset generated from 500 PC3-CRT cells, we mixed 500 cells with zebrafish larva at post hatching day $1,4,6,7,8,10$ and 12 , respectively. The total RNAs were isolated from each mixture and used for qPCR analysis. This was expected to minimize the error caused by the slightly different growth rate of zebrafish in our system. The total RNA of each mixture and the cDNA were obtained using TRIzol ${ }^{\circledR}$ reagent (Thermo Fisher Scientific) and SuperScript IV Reverse Transcriptase (Thermo Fisher Scientific), respectively, following the manufacturer's instructions. For each sample, a total amount of $1 \mu \mathrm{g}$ of RNA was used for cDNA synthesis.

Twenty microliters of cDNA was synthesized for each RNA sample and the cDNA was diluted 10-fold with DNase/RNasefree molecular water. The qPCR was performed using GoTaq DNA polymerase system (Promega, Madison, WI, USA) with SYBR-Green. Each $10 \mu 1$ reaction contained $2 \mu 1$ cDNA from each sample. Primers used for qPCR are listed in Fig. 2c. The ABI 7900HT Fast Real-Time PCR system with Fast 384-Well Block (Thermo Fisher Scientific) was used for the qPCR with the program: $2 \mathrm{~min}$ at $50^{\circ} \mathrm{C}, 2 \mathrm{~min}$ at $94^{\circ} \mathrm{C}$, and 40 cycles of 94 , 55 and $72^{\circ} \mathrm{C}$ with $30 \mathrm{sec}$ at each temperature. The raw $\mathrm{Ct}$ values of tbp and hprtl expressions (x-axis) were used to construct standard curves against the $\log \left({ }_{10}\right)$ of PC3-CTR cell numbers mixed with each fish larva (y-axis). The standard curve and the regression equation were used to estimate the number of PC3-CTR cells in each of the experimental zebrafish larval individual based on the Ct values of $t b p$ and hprtl.

Three PCa tumor cell development genetic markers; prostate cancer associated 3 gene ( $p c a 3$ ), transient receptor potential ion channel melastatin $8(\operatorname{trpm} 8)$ and prostate-specific membrane antigen ( psma). No zebrafish homologous genes for these three marker genes were found based on BLASTn analysis. These markers were only detected in human PC3-CTR cells not zebrafish larvae (Fig. 2b). All primers used for qPCR in the present study are listed in Fig. 2c.

\section{Results}

Temperature does not affect the survival of xenografted zebrafish larvae. Two temperatures were used in this study for zebrafish embryo hatching, and larval zebrafish nursery post PC3-CTR cell implantation. The temperature $\left(28^{\circ} \mathrm{C}\right)$ is close to normal zebrafish hatching and nursery temperature while $32^{\circ} \mathrm{C}$ allows better growth and development of PC3-CTR cells in the larvae based on our observations. Incubated at $32^{\circ} \mathrm{C}$, $59.2 \pm 9.0 \%$ zebrafish embryos successfully hatched, which was significantly lower $(\mathrm{P}=0.002)$ than the hatching rate of the embryos raised at $28^{\circ} \mathrm{C}(93.7 \pm 2.7 \%)$ (Fig. 1c). However, no significant difference in post $\mathrm{PC} 3-\mathrm{CTR}$ injection survival rates of zebrafish larvae was observed between the two different rearing temperatures (Fig. $1 \mathrm{~b}$ and c). Large mortalities were observed in the larvae at day 1 post PC3-CTR implantation (PID1) incubated at $28^{\circ} \mathrm{C}(24.2 \pm 4.7 \%)$ or $32^{\circ} \mathrm{C}(33.3 \pm 5.4 \%)$, but no significant difference was found between these mortality rates (Fig. 1b). Therefore, we used $32^{\circ} \mathrm{C}$ to raise the larval zebrafish in the rest of the study to better grow the PC3-CTR cells in zebrafish larvae. Occasional deaths of the larvae were observed prior to post injection day 6 (PID6). In contrast, an increase of mortality was observed starting at PID6 and lasted until almost 100\% mortality in the larvae at PID14. Very few larvae with PC3-CTR survived after PID14 (Fig. 1c).

PC3-CTR cells migrated and proliferated along with the development of zebrafish larvae. The PC3-CTR cells were labeled with Qtracker ${ }^{\circledR} 525$ prior to the implantation into the larval zebrafish. Fluorescent microscopy was used to track the PC3-CTR cells injected into the larvae. At PID1, a small number of cells with Qtracker 525 signals (green) were located near the injection site of the PC3-CTR cells (Fig. 3a). IF staining with human nuclei antibody (red) also demonstrated the limited distribution of PC3-CTR cells in the larvae (Fig. 3b). No obvious migration of PC3-CTR cells in larval zebrafish was observed at PID1. Signals of Qtracker 525 in live PC3-CTR cells were hard to detect after PID3, therefore, IF staining of human nuclei was used to detect the distributions of PC3-CTR cells in larval fish. PC3-CTR cells were found widely distributed at the posterior section of the fish (between original PC3-CTR injection site and the tail of the larva; Fig. 3c). PC3-CTR cells were also detected in the anterior section of the fish (between head and original PC3-CTR injection site, Fig. 3c), but the number was limited. The number of PC3-CTR cells in larval zebrafish was markedly elevated from PID5 on. PC3-CTR cells were located from head to tail and colonized a large area in the head of the larva (Fig. 3d). At PID7, more PC3-CTR cells were observed in the head area while less PC3-CTR cells were seen at the posterior section of the larva (Fig. 3e).

PC3-CTR cells in zebrafish larvae demonstrated rapid proliferation. To estimate the number of PC3-CTR cells in each zebrafish larva, qPCR targeting PC3-CTR housekeeping genes, $t b p$ and hprtl, was used to generate cell-number/ $\mathrm{Ct}$-value curves. The number of PC3-CTR mixed with each zebrafish larva was exponentially correlated with the $\mathrm{Ct}$ values of $t b p\left(\mathrm{Ct}_{t b p}\right)$ and hprtl $\left(\mathrm{Ct}_{h p r t l}\right)$, although the regression equations for these two curves were different. With the curve created from $t b p$ gene expression, an equation $y=5 E+10 e^{-0.646 x}$ was generated to calculate the number of PC3-CTR cells in any given zebrafish larva with a Ct value of $t b p$ expression (Fig. 4a). The equation generated with the Ct values of hprtl expression was $y=3 E+15 e^{-1.217 x}$ (Fig. 4b). Larval zebrafish implanted with PC3-CTR cells were harvested at PID2, 4, 6, and 8. The number of PC3-CTR cells in each larval individual was estimated using the two equations. For example, the $\mathrm{Ct}_{t b p}$ of a zebrafish larva at PID2 was $32.85(\mathrm{x}=32.85)$. Using the equation, $y=5 E+10 e^{-0.646 x}$, we calculated the total number of PC3-CTR in the larva was 30 . With the cDNA from the same larval individual, we obtained the $\mathrm{Ct}_{h p r t l}=26.45$. Using the second equation, 


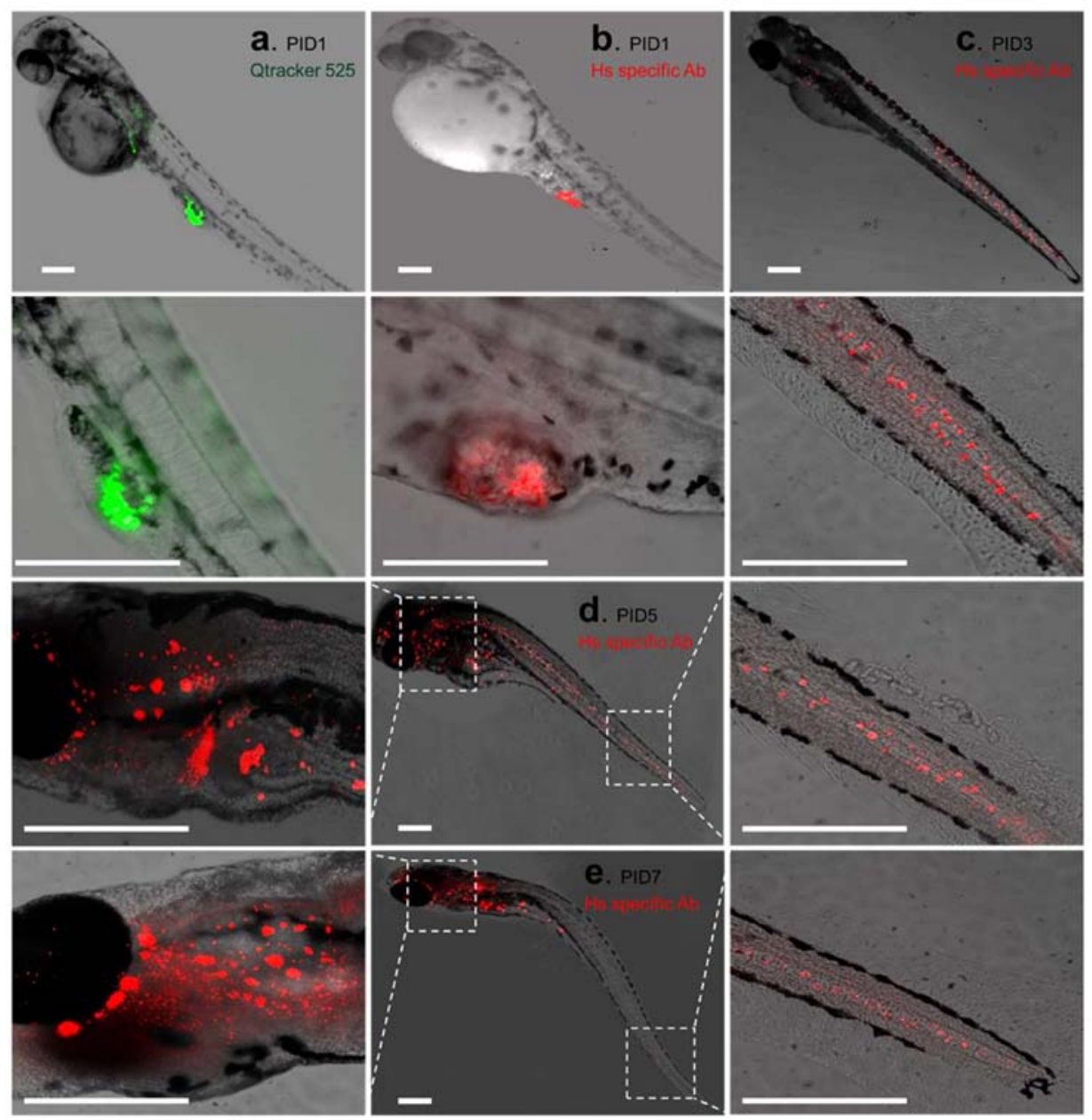

Figure 3. Migration and proliferation of PC3-CRT cells in vivo. (a) The PC3-CRT cells at PID1 were tracked by the live cell tracking dye Qtracker 525 (green). (b) The PC3-CRT cells in vivo were monitored by immunofluorescent staining by human nucleus specific antibody with Alexa 594 labeled secondary antibody (red). (c) PC3-CRT cell migration and proliferation in vivo at PID3. (d) Signals for PC3-CRT cells in vivo at PID5. Higher magnifications were used to visualize the detailed distributions of PC3-CRT cells at anterior (left) and posterior (right) sections. (e) Distribution of PC3 cells at PID7 in vivo, with higher magnification at anterior (left) and posterior (right) sections. Scale bar, $200 \mu \mathrm{m}$.

$y=3 E+15 e^{-1.217 x}$, we concluded the number of PC3-CTR cells in this larva equals 27. The number of PC3-CTR cells in each larval individual at each PID point was estimated using the two equations.

Two graphs showing the changes of PC3-CTR cells in zebrafish larvae during development were constructed (Fig. 4c and d). The estimated numbers of PC3-CTR cells in each larva using different equations demonstrated were similar. At PID2, the average number of PC3-CTR cells in each larva was 33 based on the $\mathrm{Ct}_{\mathrm{tbp}}$ values. Using the $\mathrm{Ct}_{\text {hprtl }}$ based quantification method, the average number of PC3-CTR cells in each individual was 30 . The two PC3-CTR number estimation systems also demonstrated high consistencies in the larvae at other time-points (Fig. $4 \mathrm{c}$ and d). Consistent to the observations of PC3-CTR cell populations in zebrafish larvae, rapid expansions of PC3-CTR cells were also identified using the qPCR quantification assays. At PID4, the numbers of PC3-CTR cells estimated by the two equations increased to 93 and 158, respectively, in larval zebrafish. By PID6, over 1,000 PC3-CTR cells were estimated in each larva $(1,624$ by equation 1 vs. 1,013 by equation 2). At the final time-point tested in this study (PID6), the average numbers of in vivo PC3-CTR cells in fish reached, respectively 2,004 and 2,440 according to the results from the two equations. With the PC3-CTR numbers in each larva estimated by $\mathrm{Ct}$ values of $h p r t 1\left(\mathrm{Y}_{\mathrm{HPRT1}}\right)$ and $t b p\left(\mathrm{Y}_{\mathrm{TвP}}\right)$, we also generated a graph demonstrating the ratios of the two estimated numbers $\left(\mathrm{Y}_{\mathrm{HPRT}} / \mathrm{Y}_{\mathrm{TBP}}\right)$. At day 2 and day 6 post PCa cell xenograft, the number of PC3-CTR cells estimated by hprt 1 and $t b p$ models was very close with the ratios at 0.999 and 0.959 , respectively (Fig. 4e). At PID 4 and 8, the number of in vivo PC3-CTR cells estimated with the hprtl derived equation was higher than those calculated from tbp derived formula. The values of $\mathrm{Y}_{\mathrm{HPRT}} / \mathrm{Y}_{\mathrm{TBP}}$ at PID 4 and 8 were 1.78 and 2.44, respectively (Fig. 4e).

PC3-CTR demonstrated elevated aggressiveness in vivo. Three commonly used PCa cell markers, pca3, trpm 8 and psma, were used to evaluate the development and aggressiveness of PC3-CTR cells (24-26) in larval zebrafish. The expressions of these three molecular markers along the development of zebrafish larvae showed similar patterns. Increase of pca3 expression in PC3-CTR implanted larvae was detected from PID2 to PID6 compared to the pca3 level in PC3-CTR cells in vitro. The expression of pca3 showed the greatest upregulation in the larvae at PID6, which was 6.90 1 1.34-fold greater than that in control PC3-CTR cells 

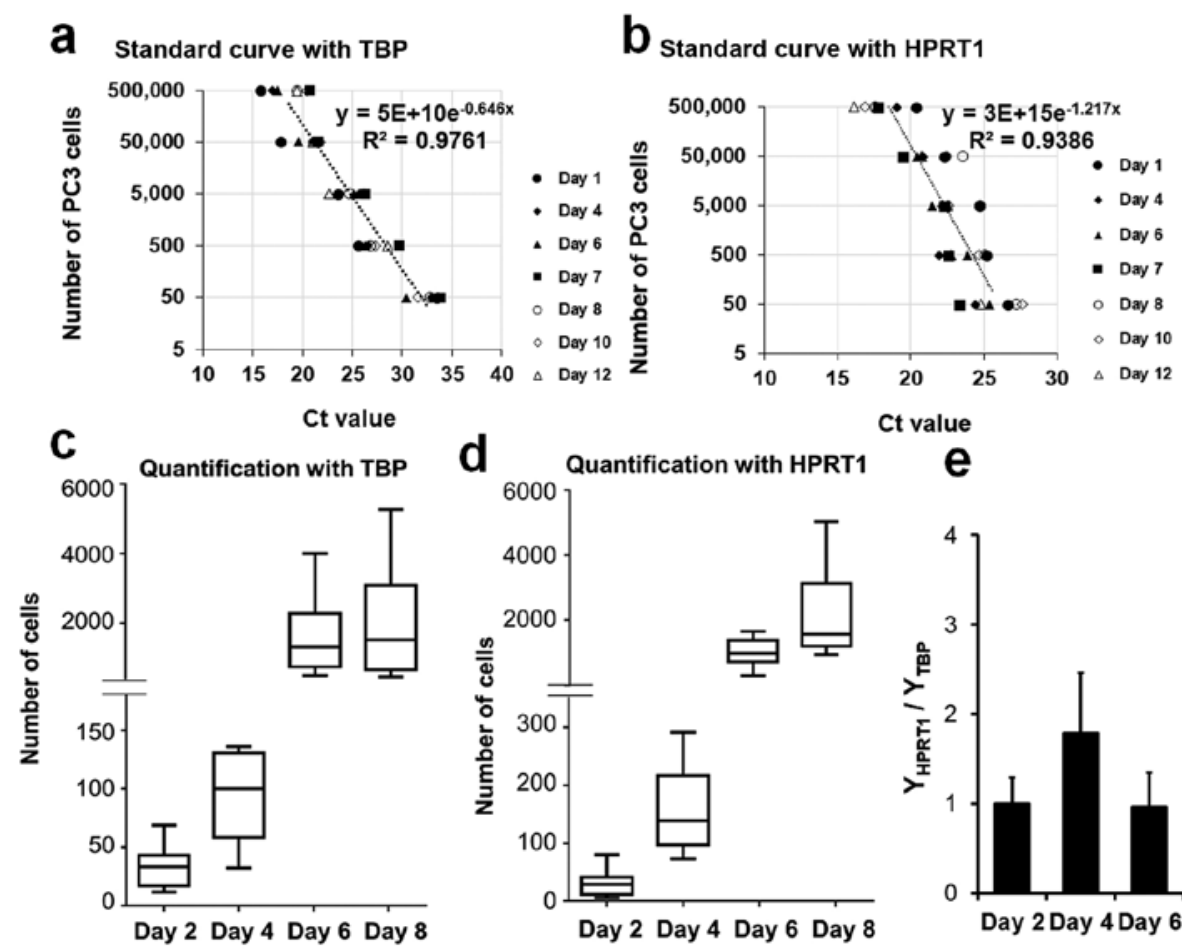

e
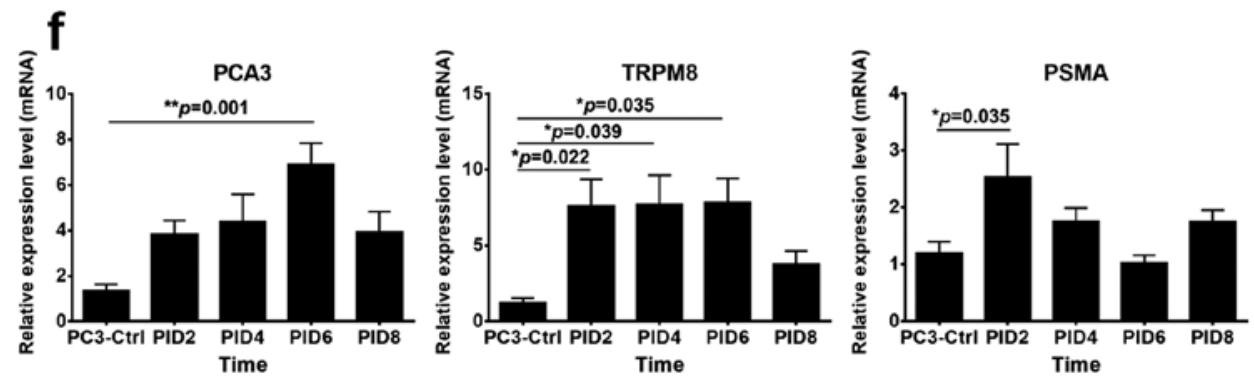

Figure 4. Quantification of PC3-CRT cells in zebrafish larvae with qPCR. (a) The housekeeping gene tbp was used to estimate the number of PC3-CRT cells in each injected zebrafish larva. The standard curve with the $\log _{10}(\mathrm{Ct})$ values of $t b p$ expression and PC3-CRT cell numbers was created and the equation for regression was generated $(\mathrm{n}=5-8)$. (b) The standard curve was also generated with $\log _{10}(\mathrm{Ct})$ values of the other housekeeping gene hprt1. (c) The estimated PC3-CRT cell numbers in each zebrafish larval individual calculated by the regression equation generated from $t b p$ standard curve. (d) The number of PC3-CRT cells in each PC3-CRT injected zebrafish larva was also estimated with hprt1 standard curve. (e) Comparisons of the numbers of in vivo PC3-CRT cells calculated with different formula. $\mathrm{Y}_{\mathrm{hprt}}$, cell numbers calculated with equation $y=3 E+15 e^{-1.217 x}$; $\mathrm{Y}_{\mathrm{tbp}}$, cell numbers calculated with equation $y=3 E+10 e^{-0.646 x} . \mathrm{Y}_{\mathrm{hprt} 1} / \mathrm{Y}_{\mathrm{tbp}}$, the ratio of estimated PC3-CRT cell numbers for each sample. (f) Relative mRNA levels of pca3, trpm8 and psma were estimated using qPCR along the time course of zebrafish larval development ( $\mathrm{n}=6-12$ at each time-point).

$(\mathrm{P}=0.001$; Fig. 4f). Similarly, expression of trpm8 was significantly upregulated at PID2 in PC3-CTR cell implanted larvae by $7.63 \pm 2.07(\mathrm{P}=0.022)$ compared to $\operatorname{trpm} 8$ expression in PC3-CTR in vitro. This upregulation sustained from PID2 to PID6 with >7-fold difference at each time-point (Fig. 4f). The transcriptome production of psma in PC3-CTR cells in vivo demonstrated a rapid elevation at PID2, with a 2.54 \pm 0.51 -fold increase compared to in vitro PC3-CTR cells. However, the upregulation of psma in PC3-CTR cell implanted larvae was attenuated from PID4 with no significant difference than that in PC3-CTR cells in vitro (Fig. 4f). In summary, the expression profiles of these PCa cell markers suggested that the progression and invasiveness of the PC3-CTR cells were rapidly enhanced when implanted into larval zebrafish.

\section{Discussion}

Present results demonstrate that the human PCa cell zebrafish xenograft model provides a rapid method for the analyses of cancer cell migration and metastatic process in vivo. Comparisons of human cancer xenograft models have been made between zebrafish and nude mice (nu/nu). PCa cell line, DU145, injected to mice through the lateral tail vein, did not demonstrate metastasis until three months post injection. In contrast, metastasis of DU145 cells was observed in zebrafish larvae within $60 \mathrm{~h}$ post implantation (27). Based on our previous trials, the development of PC3-CTR cells varies with the different nursing conditions. Other than the post cell injection nursing temperature, $32^{\circ} \mathrm{C}$ used in this study, we also applied lower $\left(28^{\circ} \mathrm{C}\right)$ or higher $\left(36^{\circ} \mathrm{C}\right)$ temperatures in some trials. The development of PC3-CTR cells in larval zebrafish was significantly inhibited, which was reflected by the migrating and proliferating rates of the cells (based on observation). In contrast, no observations were made in the larval zebrafish cultured at $36^{\circ} \mathrm{C}$ because of the high mortalities, although $36^{\circ} \mathrm{C}$ is the best temperature for PC3-CTR cells grown in vitro compared to the other temperatures. We also tempted to optimize this model by using the larval 
zebrafish at different development stages, such as at post fertilization day (pfd) 4 or 6 . However, our observation on PC3-CTR cells injected into fish larvae at pfd 4 or 6 showed a severely inhibited proliferation. This was likely caused by the enhanced host immune system in zebrafish larvae with the development $(11,28)$. No larval zebrafish with implanted PC3-CRT cells survived beyond PID10. Similar results were reported from previous studies. Implantation of wild-type PC3 cells $(17,29)$ and primarily isolated prostate tumor inducing cells (17) with less aggressiveness compared to PC3-CRT cells (18). The adverse effects caused by the rapid tumor cell proliferation could not be overcome by the host. An improved zebrafish PCa cell xenograft model with a longer survival period is desired for application in PCa diagnosis.

The external environmental conditions for PCa cells grown in vivo are important factors for cell development. However, the fate of implanted cells is primarily determined by the cell type. In the present study, we used the PC3-CTR cell, which is a modified cell line based on popular cell line PC 3 established from bone metastasis of prostate cancer in 1979 (30). Because of the high tumorigenicity of this cell line, the PC3-CTR cells implanted in larval zebrafish demonstrated a high aggressiveness. The proliferating rate of PC3-CTR in vivo was high. For each larval individual, in this study, 5-6 PC3-CTR cells were initially injected subcutaneously. Approximately 2,000 cells were found in the individual at PID8 and they were widely distributed in the larva, which suggested an active migration in vivo. Compared to PC3-CTR cells, other PCa cell lines, DU145 and LNCaP, used in previous studies, showed slightly different behavior in zebrafish larvae. Migrations of DU145 and $\mathrm{LNCaP}$ cells in vivo were observed $30 \mathrm{~h}$ post injection (hpi) with the colonization established across the whole body (27). In another earlier study, tumor-initiating cells (TICs) were isolated from prostates of PCa patients and implanted to zebrafish larvae. Although the TICs demonstrated a strong ability in migration and proliferation in vitro, no aggressive behavior of TICs inside zebrafish larvae were observed. Cell migration was observed towards the posterior section of body trunk with very limited expansion of the colony in vivo (17). The difference in cell behavior was likely caused by different tumorigenesity of TICs and PCa cell lines.

The PCa cells used in different studies demonstrated different proliferating levels in vivo despite all these cells exhibiting extraordinary proliferating abilities in vitro $(17,27,31)$. This was primarily caused by the different cell types but also attributed to the methods of quantification. Since the PCa cells were all fluorescently labeled prior to implantation, one of the commonly used methods for quantification is direct counting with cell imaging. Because of the high transparency of larval zebrafish body trunk, it is possible to visualize the signals given by the pre-labeled cells using fluorescent microscopy (27). The imaging analysis software ImageJ (32) provided a powerful tool for cell counting with no cost. However, the accuracy of cell counting is primarily determined by technique of microscopy. Most cell counting studies of xenograft cells were based on the 2-D images, which were not able to precisely differentiate the cells distributed in three dimensions. Therefore, the signals detected by microscopy could not represent all positive cells in vivo. Although more advanced microscopic techniques, such as confocal microscopy $(33,34)$ and 3-D imaging $(35,36)$, were applied in tumor cell caption and they also increased the expenses and complexities of cell quantification methods. The cell quantification method used in this study is based on qPCR technique using the expression profiles of housekeeping genes in PC3 cells. This technique took the advantage of high sensitivity of PCR assay. Moreover, the specificity was also improved by the carefully designed and fully characterized primers. The selection of housekeeping genes for PCa cells was based on previous studies. The genes, tbp and hprtl, were all previously used for housekeeping genes in PCa studies $(22,37,38)$, and demonstrated great reliability in quantification of gene expression. Other previously reported housekeeping genes, including gapdh, actb and 18S rRNA, were also tested in this study. Based on the sensitivity and linear regression analysis of the $\mathrm{Ct}$ values of the housekeeping genes, we identified $t b p$ and hprtl as the best candidate housekeeping genes for this study. Similar techniques were also used in quantification of human tumor cell xenografts in mice $(39,40)$. We also considered the potential errors during the operation for in vivo $\mathrm{PCa}$ cell quantification with qPCR. The standard curves of PCa cell quantification were generated with $\mathrm{Ct}$ values of housekeeping genes from a mixture of in vitro cultured PC3-CRT cells and larval zebrafish. We used these curves to estimate the number of PC3-CRT cells in vivo. The characteristics of PC3-CRT cells might be affected by the surrounding environment, which could possibly alter the expression patterns of housekeeping genes. However, these selected housekeeping genes of PCa cells have been previously confirmed to be constitutively expressed under effects of various conditions. Therefore, we expected very minor influence on the quantification of PC3-CRT cells in vivo. Compared to conventional cell imaging analysis, this assay provided a rapid method for xenograft cell quantification with minimum requirement for equipment.

The three PCa cell markers, pca3, trpm 8 and psma, were used as references for invasiveness of PC3-CTR cells in vivo. The pca3, also known as differential display code 3 (DD3), is a prostate-specific non-coding mRNA, which is overexpressed in tumor cells (41). The cells with overexpression of pca 3 in prostate is often associated with a greater tumor aggressiveness $(42,43)$. As a member of transient receptor potential (TRP) ion channel family, the TRPM8 is involved in a number of prostate tumor cell activities, such as proliferation $(44,45)$, apoptosis $(46)$ and cell migration $(45,47)$. Because of its specificity and roles in prostate tumor cell progression, trpm8 has been considered to be a potential target for diagnostic strategies, and pharmaceutical treatment of PCas (48). The PSMA is a prostate specific membrane antigen that has been correlated with a number of prostate aggressive disease, including PCas (49). More than 10-fold expression levels of this molecule has been detected in many states of PCas (26). The basal expression level of psma in PC 3 cells was reported to be low $(26,50)$, although we detected the expression of this gene in mRNA level in PC3-CTR cells. Our finding suggested that the expression levels of these three PCa markers were all elevated with the development of PC3-CTR cells in vivo, which is positively correlated with the increase of PC3-CTR cell proliferation and migration based on the result of observation and quantification. 
Our results indicated that the in vivo experiment of larval zebrafish was ideal for PC3-CTR cell progression. The development of PCa cells in zebrafish larvae appeared to be significantly accelerated compared to that in mammalian models (several days in fish vs. several months in mice). However, the rapid development of PC3-CTR cells demonstrated lethal effects to zebrafish larvae. Large mortalities were observed after PID5 and very few individuals with implanted PC3-CTR cells survived beyond PID14. The rapidly grown PC3-CTR cells appeared to heavily impact the normal growth of the larvae. The average growth rates of PC3-CTR implanted larvae were markedly lower than control larvae without implanted PC3-CTR cells. The tumor burden was likely the cause of nutritional deficiency in the host (51). Therefore, an improved nutritional condition is necessary to maintain the growth of zebrafish larvae during the experiment.

In summary, the present studies demonstrate the utility of zebra fish model to assess tumorigenicity and aggressiveness of prostate cancer cell lines; and the model can be applied for quick assessment of aggressiveness of primary prostate cancer cells derived from patient biopsies.

\section{Acknowledgements}

The present study is supported by the Louisiana Campus Research Initiatives, and the NIH grant CA-096534 to G.S.

\section{References}

1. American Cancer Society: What are the key statistics about prostate cancer? 2015.

2. van Leeuwen PJ, Connolly D, Gavin A, Roobol MJ, Black A, Bangma $\mathrm{CH}$ and Schröder FH: Prostate cancer mortality in screen and clinically detected prostate cancer: Estimating the screening benefit. Eur J Cancer 46: 377-383, 2010.

3. Rajasekhar VK, Studer L, Gerald W, Socci ND and Scher HI: Tumour-initiating stem-like cells in human prostate cancer exhibit increased NF- $\kappa B$ signalling. Nat Commun 2: 162, 2011.

4. Collins AT, Berry PA, Hyde C, Stower MJ and Maitland NJ: Prospective identification of tumorigenic prostate cancer stem cells. Cancer Res 65: 10946-10951, 2005.

5. Chen Y, Zhao J, Luo Y, Wang Y, Wei N and Jiang Y: Isolation and identification of cancer stem-like cells from side population of human prostate cancer cells. J Huazhong Univ Sci Technolog Med Sci 32: 697-703, 2012.

6. Bokhorst LP, Bangma CH, van Leenders GJ, Lous JJ, Moss SM Schröder FH and Roobol MJ: Prostate-specific antigen-based prostate cancer screening: reduction of prostate cancer mortality after correction for nonattendance and contamination in the Rotterdam section of the European Randomized Study of Screening for Prostate Cancer. Eur Urol 65: 329-336, 2013.

7. Shah G: A human cancer cell specific gene product. USPTO (ed.) Girish V. Shah, 2011.

8. Park SI, Kim SJ, McCauley LK and Gallick GE: Pre-clinical mouse models of human prostate cancer and their utility in drug discovery. Curr Protoc Pharmacol Chapter 14: Unit 14.15, 2010.

9. Hafeez BB, Zhong W, Fischer JW, Mustafa A, Shi X, Meske L, Hong H, Cai W, Havighurst T, Kim K, et al: Plumbagin, a medicinal plant (Plumbago zeylanica)-derived 1,4-naphthoquinone, inhibits growth and metastasis of human prostate cancer PC-3M-luciferase cells in an orthotopic xenograft mouse model. Mol Oncol 7: 428-439, 2013.

10. Hansen AG, Arnold SA, Jiang M, Palmer TD, Ketova T, Merkel A, Pickup M, Samaras S, Shyr Y, Moses HL, et al: ALCAM/CD166 is a TGF- $\beta$-responsive marker and functional regulator of prostate cancer metastasis to bone. Cancer Res 74 1404-1415, 2014.

11. Moshal KS, Ferri-Lagneau KF and Leung T: Zebrafish model: Worth considering in defining tumor angiogenesis. Trends Cardiovasc Med 20: 114-119, 2010.
12. Liu S and Leach SD: Zebrafish models for cancer. Annu Rev Pathol 6: 71-93, 2011.

13. Tat J,Liu M and Wen XY: Zebrafish cancer and metastasis models for in vivo drug discovery. Drug Discov Today Technol 10: e83-e89, 2013.

14. Yen J, White RM and Stemple DL: Zebrafish models of cancer: Progress and future challenges. Curr Opin Genet Dev 24: 38-45, 2014.

15. Patton EE and Zon LI: Taking human cancer genes to the fish: A transgenic model of melanoma in zebrafish. Zebrafish 1: 363-368, 2005.

16. White RM, Sessa A, Burke C, Bowman T, LeBlanc J, Ceol C, Bourque C, Dovey M, Goessling W, Burns CE, et al: Transparent adult zebrafish as a tool for in vivo transplantation analysis. Cell Stem Cell 2: 183-189, 2008.

17. Bansal N, Davis S, Tereshchenko I, Budak-Alpdogan T, Zhong H, Stein MN, Kim IY, Dipaola RS, Bertino JR and Sabaawy HE: Enrichment of human prostate cancer cells with tumor initiating properties in mouse and zebrafish xenografts by differential adhesion. Prostate 74: 187-200, 2014.

18. Shah GV, Muralidharan A, Gokulgandhi M, Soan K and Thomas S: Cadherin switching and activation of beta-catenin signaling underlie proinvasive actions of calcitonin-calcitonin receptor axis in prostate cancer. J Biol Chem 284: 1018-1030, 2009.

19. Westerfield M: The zebrafish book. A guide for the laboratory use of zebrafish (Danio rerio). 5th edition. University of Oregon Press, Eugene, OR, 2007.

20. Schmidt U, Fuessel S, Koch R, Baretton GB, Lohse A, Tomasetti S, Unversucht S, Froehner M, Wirth MP and Meye A: Quantitative multi-gene expression profiling of primary prostate cancer. Prostate 66: 1521-1534, 2006.

21. Valente V, Teixeira SA, Neder L, Okamoto OK, Oba-Shinjo SM, Marie SK, Scrideli CA, Paçó-Larson ML and Carlotti CG Jr: Selection of suitable housekeeping genes for expression analysis in glioblastoma using quantitative RT-PCR. BMC Mol Biol 10: $17,2009$.

22. Ohl F, Jung M, Xu C, Stephan C, Rabien A, Burkhardt M, Nitsche A, Kristiansen G, Loening SA, Radonić A, et al: Gene expression studies in prostate cancer tissue: Which reference gene should be selected for normalization? J Mol Med (Berl) 83: 1014-1024, 2005.

23. Altschul SF, Gish W, Miller W, Myers EW and Lipman DJ: Basic local alignment search tool. J Mol Biol 215: 403-410, 1990.

24. Ferreira LB, Palumbo A, de Mello KD, Sternberg C, Caetano MS, de Oliveira FL, Neves AF, Nasciutti LE, Goulart LR and Gimba ER: PCA3 noncoding RNA is involved in the control of prostate-cancer cell survival and modulates androgen receptor signaling. BMC Cancer 12: 507, 2012.

25. Bai VU, Murthy S, Chinnakannu K, Muhletaler F, Tejwani S, Barrack ER, Kim SH, Menon M and Veer Reddy GP: Androgen regulated TRPM8 expression: A potential mRNA marker for metastatic prostate cancer detection in body fluids. Int J Oncol 36: 443-450, 2010.

26. Ghosh A, Wang X, Klein E and Heston WD: Novel role of prostate-specific membrane antigen in suppressing prostate cancer invasiveness. Cancer Res 65: 727-731, 2005.

27. Teng Y, Xie X, Walker S, White DT, Mumm JS and Cowell JK: Evaluating human cancer cell metastasis in zebrafish. BMC Cancer 13: 453, 2013.

28. Taylor AM and Zon LI: Zebrafish tumor assays: The state of transplantation. Zebrafish 6: 339-346, 2009.

29. Spaink HP, Cui C, Wiweger MI, Jansen HJ, Veneman WJ, Marín-Juez R, de Sonneville J, Ordas A, Torraca V, van der Ent W, et al: Robotic injection of zebrafish embryos for highthroughput screening in disease models. Methods 62: 246-254, 2013.

30. Kaighn ME, Narayan KS, Ohnuki Y, Lechner JF and Jones LW: Establishment and characterization of a human prostatic carcinoma cell line (PC-3). Invest Urol 17: 16-23, 1979.

31. Abasolo I, Yang L, Haleem R, Xiao W, Pio R, Cuttitta F, Montuenga LM, Kozlowski JM, Calvo A and Wang Z: Overexpression of adrenomedullin gene markedly inhibits proliferation of PC3 prostate cancer cells in vitro and in vivo. Mol Cell Endocrinol 199: 179-187, 2003.

32. Schneider CA, Rasband WS and Eliceiri KW: NIH Image to ImageJ: 25 years of image analysis. Nat Methods 9: 671-675, 2012 . 
33. Allocca G, Kusumbe AP, Ramasamy SK and Wang N: Confocal/ two-photon microscopy in studying colonisation of cancer cells in bone using xenograft mouse models. Bonekey Rep 5: 851, 2016.

34. Chittajallu DR, Florian S, Kohler RH, Iwamoto Y, Orth JD, Weissleder R, Danuser G and Mitchison TJ: In vivo cell-cycle profiling in xenograft tumors by quantitative intravital microscopy. Nat Methods 12: 577-585, 2015.

35. Bocci G, Buffa F, Canu B, Concu R, Fioravanti A, Orlandi P and Pisanu T: A new biometric tool for three-dimensional subcutaneous tumor scanning in mice. In Vivo 28: 75-80, 2014.

36. Oldham M, Sakhalkar H, Oliver T, Wang YM, Kirpatrick J, Cao Y, Badea C, Johnson GA and Dewhirst M: Threedimensional imaging of xenograft tumors using optical computed and emission tomography. Med Phys 33: 3193-3202, 2006.

37. Tsaur I, Renninger M, Hennenlotter J, Oppermann E, Munz M, Kuehs U, Stenzl A and Schilling D: Reliable housekeeping gene combination for quantitative PCR of lymph nodes in patients with prostate cancer. Anticancer Res 33: 5243-5248, 2013.

38. Rho HW, Lee BC, Choi ES, Choi IJ, Lee YS and Goh SH: Identification of valid reference genes for gene expression studies of human stomach cancer by reverse transcription-qPCR. BMC Cancer 10: 240, 2010.

39. Alcoser SY, Kimmel DJ, Borgel SD, Carter JP, Dougherty KM and Hollingshead MG: Real-time PCR-based assay to quantify the relative amount of human and mouse tissue present in tumor xenografts. BMC Biotechnol 11: 124, 2011.

40. Preston Campbell J, Mulcrone P, Masood SK, Karolak M, Merkel A, Hebron K, Zijlstra A, Sterling J and Elefteriou F: TRIzol and Alu qPCR-based quantification of metastatic seeding within the skeleton. Sci Rep 5: 12635, 2015.

41. Bussemakers MJ, van Bokhoven A, Verhaegh GW, Smit FP, Karthaus HF, Schalken JA, Debruyne FM, Ru N and Isaacs WB: DD3: A new prostate-specific gene, highly overexpressed in prostate cancer. Cancer Res 59: 5975-5979, 1999.

42. Merola R, Tomao L, Antenucci A, Sperduti I, Sentinelli S, Masi S, Mandoj C, Orlandi G, Papalia R, Guaglianone S, et al: PCA3 in prostate cancer and tumor aggressiveness detection on 407 high-risk patients: A National Cancer Institute experience. J Exp Clin Cancer Res 34: 15, 2015.
43. Galasso F, Giannella R, Bruni P, Giulivo R, Barbini VR, Disanto V, Leonardi R, Pansadoro V and Sepe G: PCA3: A new tool to diagnose prostate cancer (PCa) and a guidance in biopsy decisions. Preliminary report of the UrOP study. Arch Ital Urol Androl 82: 5-9, 2010.

44. Wang Y, Wang X, Yang Z, Zhu G, Chen D and Meng Z: Menthol inhibits the proliferation and motility of prostate cancer DU145 cells. Pathol Oncol Res 18: 903-910, 2012.

45. Zhu G, Wang X, Yang Z, Cao H, Meng Z, Wang Y and Chen D: Effects of TRPM8 on the proliferation and angiogenesis of prostate cancer PC-3 cells in vivo. Oncol Lett 2: 1213-1217, 2011.

46. Wertz IE and Dixit VM: Characterization of calcium releaseactivated apoptosis of LNCaP prostate cancer cells. J Biol Chem 275: 11470-11477, 2000.

47. Gkika D, Flourakis M, Lemonnier L and Prevarskaya N: PSA reduces prostate cancer cell motility by stimulating TRPM8 activity and plasma membrane expression. Oncogene 29: 4611-4616, 2010.

48. Zhang L and Barritt GJ: TRPM8 in prostate cancer cells: A potential diagnostic and prognostic marker with a secretory function? Endocr Relat Cancer 13: 27-38, 2006.

49. Sweat SD, Pacelli A, Murphy GP and Bostwick DG: Prostatespecific membrane antigen expression is greatest in prostate adenocarcinoma and lymph node metastases. Urology 52: 637-640, 1998

50. Yao V, Berkman CE, Choi JK, O'Keefe DS and Bacich DJ: Expression of prostate-specific membrane antigen (PSMA), increases cell folate uptake and proliferation and suggests a novel role for PSMA in the uptake of the non-polyglutamated folate, folic acid. Prostate 70: 305-316, 2010.

51. Macciò A, Madeddu C, Gramignano G, Mulas C, Tanca L, Cherchi MC, Floris C, Omoto I, Barracca A and Ganz T: The role of inflammation, iron, and nutritional status in cancer-related anemia: Results of a large, prospective, observational study. Haematologica 100: 124-132, 2015. 